\title{
ELIMINATION OF RESONANCES IN CODIMENSION ONE FOLIATIONS
}

\author{
M. Fernández Duque
}

\begin{abstract}
The problem of reduction of singularities for germs of codimension one foliations in dimension three has been solved by Cano in [3]. The author divides the proof in two steps. The first one consists in getting pre-simple points and the second one is the passage from pre-simple to simple points. In arbitrary dimension of the ambient space the problem is open. In this paper we solve the second step of the problem.
\end{abstract}

2010 Mathematics Subject Classification: 32S45, 32S65, 37F75.

Key words: Singular foliations, reduction of singularities, resonances.

\section{Introduction}

Reduction of singularities for germs of holomorphic codimension one foliations in $\left(\mathbb{C}^{n}, 0\right)$ is an unsolved problem.

In ambient dimension $n=2$, the first complete proof has been given by Seidenberg in [9]. It is one of the main tools for the understanding of germs of vector fields and foliations in $\left(\mathbb{C}^{2}, 0\right)$. For instance, it is absolutely necessary to support a holonomic study of those foliations (see $[\mathbf{8}]$ ) or to prove the existence of invariant analytic curves (separatrices) (see $[\mathbf{1}]$ ).

In ambient dimension $n=3$, the problem has been first solved in the non-dicritical case $([\mathbf{2}],[\mathbf{4}])$ and the result has been used to prove the existence of germs of invariant surfaces in this case. In the general case, it has been solved in $[\mathbf{3}]$.

Although there are invariant hypersurfaces in the non-dicritical situations and higher dimension [6], the general solution of the problem of reduction of singularities for $n \geq 4$ is still widely open.

In $[4]$ and $[\mathbf{3}]$ the authors have identified two kinds of points for codimension one singular foliations in higher dimensional ambient spaces. They are the pre-simple points and the simple ones. Reduction of singularities consists in obtaining at most simple points after a finite sequence of blow-ups with non singular invariant centers. To get pre-simple points 
is the first step in that problem, and the passage from pre-simple to simple points is the second and last step. In fact, pre-simple points are defined in terms of upper-semicontinuous invariants such as the multiplicity and the transversality positions of the initial forms. The definition of simple points adds non-resonance requirements to the conditions defining pre-simple points. This paper is devoted to a global solution of the second step of reduction of singularities.

The first example of a pre-simple but not simple point is the radial vector field, or equivalently, the foliation defined by

$$
\frac{d x}{x}-\frac{d y}{y}=0 .
$$

After a first dicritical blow-up we obtain simple points (in this particular case even non-singular points). Precise definitions of the notions are given below.

Up to some considerations about the Jordan Block singularities, the second step of the problem consists in the elimination of the resonances appearing on the residual vectors of the pre-simple singularities.

In order to do that in a complete global way, we construct a Control Variety $X$ with a normal crossings divisor $E$. We consider some divisors whose support is contained in $E$ in such a way that the problem of eliminating the resonances is solved once we have eliminated the indeterminacies of these divisors (in the sense of Subsection 7.2).

We end the paper by showing how to eliminate the indeterminacies of a divisor by means of a combinatorial game. In this way we complete the passage from pre-simple to simple points by means of blow-ups with invariant centers of codimension two.

\section{Preliminaries}

Contents of this section can be essentially found in $[\mathbf{3}]$.

Let $M$ be a germ around a non singular compact analytic subset of a complex analytic variety of dimension $n$. Given a point $P \in M$ we denote by $\mathcal{O}_{M, P}$ the ring of analytic functions at $P$ and by $\mathfrak{M}_{M, P}$ its

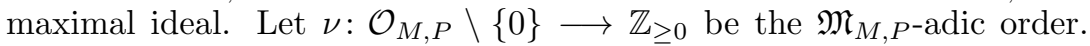
Given $a \in \mathfrak{M}_{M, P}$ we denote by $L a:=a+\mathfrak{M}_{M, P}^{2}$ its linear part.

A holomorphic singular codimension one foliation $\mathcal{F}$ of $M$ (for short, a foliation of $M$ ) is given locally at each point $P \in M$ by an equation $\Omega=0$ where

$$
\Omega=\sum_{i=1}^{n} f_{i}(\boldsymbol{x}) d x_{i} ; \quad f_{i} \in \mathcal{O}_{M, P}
$$


is an integrable 1-form germ whose coefficients have no common factors. Such an $\Omega$ is called a local generator of $\mathcal{F}$ at $P$.

Remark 1. Recall that a 1-form $\eta$ is called integrable if $\eta \wedge d \eta=0$.

Remark 2. Let $\Omega$ be a local generator of $\mathcal{F}$ at $P$ and $h$ a unit of $\mathcal{O}_{M, P}$. Then $h \cdot \Omega$ is also a local generator of $\mathcal{F}$ at $P$.

Remark 3. Any meromorphic integrable 1-form $\omega$ gives locally a unique foliation that is also denoted by $(\omega=0)$. To see this we consider a local generator $\Omega$ where $\Omega=f / g \cdot \omega$ for an appropriate meromorphic germ $f / g$. The point of multiplication by $f / g$ is to achieve that all coefficients of $\Omega$ are holomorphic functions having no common factors.

The singular locus of $\mathcal{F}$ is the subset locally given by

$$
\operatorname{Sing} \mathcal{F}=\left(f_{1}=\cdots=f_{n}=0\right) \text {. }
$$

Since the coefficients of $\omega$ have no common factors, the codimension of Sing $\mathcal{F}$ is at least 2 . If

$$
\nu\left(f_{1}, \ldots, f_{n}\right)=0
$$

we say that the foliation $\mathcal{F}$ is regular at $P$. In this case there are local coordinates such that $\mathcal{F}$ is generated by $\omega=d x_{1}^{\prime}$.

Definition 1. Let $\mathcal{L}=\left\{Y_{1}, \ldots, Y_{s}\right\}$ be a finite list of analytic irreducible subsets $Y_{j} \subset M$. We say that $\mathcal{L}$ has normal crossings at a point $P \in M$ if there are local coordinates $\left(x_{1}, \ldots, x_{n}\right)$ such that

$$
Y_{j}=\bigcap_{i \in A_{j}}\left(x_{i}=0\right), \quad j=1, \ldots, s,
$$

locally at $P$, where $A_{j} \subset\{1, \ldots, n\}$ for $j=1, \ldots, s$.

A finite list of analytic subsets has normal crossings if and only if the list whose elements are the irreducible components of these subsets has normal crossings.

Definition 2. Let $H_{1}, \ldots, H_{t}$ be hypersurfaces of $M$. We say that $H=\cup_{i=1}^{t} H_{i}$ is a normal crossings divisor if:

- each hypersurface $H_{i}$ is non singular,

- the list $\mathcal{L}=\left\{H_{1}, \ldots, H_{t}\right\}$ has normal crossings at every point of $M$.

Consider a normal crossings divisor $D \subset M$. Since $M$ is a germ around a compact, $D$ has finitely many irreducible components. At each 
point $P \in M$, there are local coordinates $\left(x_{1}, \ldots, x_{n}\right)$ such that $D$ is locally given by

$$
D=\left(\prod_{i \in A} x_{i}=0\right)
$$

These coordinates are called adapted to $D$.

The foliation $\mathcal{F}$ of $M$ can always be given locally at each point $P \in M$ by an equation $\omega=0$ where

$$
\omega=\sum_{i \in A} a_{i} \frac{d x_{i}}{x_{i}}+\sum_{i \notin A} a_{i} d x_{i} ; \quad a_{i} \in \mathcal{O}_{M, P}
$$

is an integrable 1-form whose coefficients have no common factors. Such an $\omega$ is called a local generator of $\mathcal{F}$ adapted to $D$.

Definition 3. Let $H \subset M$ be a hypersurface given locally at each point $P$ by a reduced equation $f=0, f \in \mathcal{O}_{M, P}$. We say that $H$ is an invariant hypersurface of $\mathcal{F}$ if $f$ divides $\Omega \wedge d f$, where $\Omega$ is a local generator of $\mathcal{F}$ at $P$.

The invariant components of $D$ are also called non-dicritical components. The dicritical components are then generically transversal to $\mathcal{F}$. Denote by $D_{\text {nd }}$ the union of the non-dicritical components of $D$ and by $D_{\text {dic }}$ the union of the dicritical ones. We have $D=D_{\text {nd }} \cup D_{\text {dic }}$ and locally at a point $P$ we write

$$
D=\left(\prod_{i \in A} x_{i}=0\right) ; D_{\mathrm{nd}}=\left(\prod_{i \in A_{\mathrm{nd}}} x_{i}=0\right) ; D_{\mathrm{dic}}=\left(\prod_{i \in A_{\mathrm{dic}}} x_{i}=0\right)
$$

where $A=A_{\text {nd }} \cup A_{\text {nd }}$. Note that $\left(x_{i}=0\right)$ is dicritical if and only if $x_{i}$ divides $a_{i}$. With this notation we can write equation (2) as follows:

$$
\omega=\sum_{i \in A_{\mathrm{nd}}} a_{i} \frac{d x_{i}}{x_{i}}+\sum_{i \in A_{\mathrm{dic}}} b_{i} x_{i} \frac{d x_{i}}{x_{i}}+\sum_{i \notin A} b_{i} d x_{i} ; \quad a_{i}, b_{i} \in \mathcal{O}_{M, P},
$$

where $b_{i}=a_{i} / x_{i}$ if $i \in A_{\mathrm{dic}}, b_{i}=a_{i}$ if $i \notin A$.

Definition 4. Let $\mathcal{F}$ be a foliation of $M, D$ a normal crossings divisor, $P \in M$ and $\omega$ an adapted local generator of $\mathcal{F}$ as in (2). The point $P$ is pre-simple for $(\mathcal{F}, D)$ if it satisfies one of the following conditions:

I. $\nu\left(a_{1}, \ldots, a_{n}\right)=0$.

II. There exists $i \in A$ such that the linear part $L a_{i}$ is linearly independent of $\left\{L x_{j}: j \in A\right\}$. Equivalently,

$$
a_{i} \not \equiv 0 \quad \bmod \left(\left(x_{j} \mid j \in A\right)+\mathfrak{M}_{M, P}^{2}\right) .
$$


Remark 4. If $P$ is pre-simple for $(\mathcal{F}, D)$ then it is also pre-simple for $\left(\mathcal{F}, D_{\text {nd }}\right)$. This follows directly from equation $(3)$. In fact, if $x_{i}=0$ is dicritical then $a_{i}=x_{i} b_{i}$, so $\nu\left(a_{i}\right) \geq 1$ and $a_{i} \equiv 0 \bmod \left(x_{i} \mid i \in\right.$ A) $+\mathfrak{M}_{M, P}^{2}$.

A vector field $\xi \in T_{P} M$ is tangent to $\mathcal{F}$ at $P$ if $\omega(\xi)=0$. If $\xi$ is a regular vector field tangent to $\mathcal{F}$ at $P$, we say that $\xi$ trivializes $\mathcal{F}$ at $P$.

Definition 5. The dimensional type $\tau$ of $\mathcal{F}$ at $P$ is $\tau:=n-k$, where $k$ is the dimension of the $\mathbb{C}$-vector space spanned by the tangent vectors $\xi(P)$, $\xi$ being a germ of vector field tangent to $\mathcal{F}$.

If $\tau$ is the dimensional type of $\mathcal{F}$ at $P$, as a consequence of the rectification theorem of vector fields, there are local coordinates such that $\mathcal{F}$ is given by a local equation $\omega=0$ where $\omega$ has the form

$$
\omega=\sum_{i=1}^{\tau} a_{i}\left(x_{1}, \ldots, x_{\tau}\right) d x_{i} .
$$

Proposition 1. The dimensional type is upper semicontinuous.

Proof: Let $P$ be a point of dimensional type $\tau$. By definition there are $n-\tau$ trivializing vector fields independent at $P$. These vector fields are independent at the points of an open neighborhood $U$. Therefore, the points of $U$ have at most dimensional type $\tau$.

Proposition 2. Let $e$ be the number of non-dicritical components of $D$ passing through a pre-simple point $P$. We have that

$$
e \leq \tau \leq e+1
$$

Proof: Take the notation as in equation (3). If we are in case I of Definition 4, there are two options:

- There is $i \in A_{\text {nd }}$ with $a_{i}$ unit. In this case the vector fields

$$
\xi_{k}=x_{i} b_{k} \frac{\partial}{\partial x_{i}}-a_{i} \frac{\partial}{\partial x_{k}}, \text { for } k \notin A_{\mathrm{nd}},
$$

trivialize $\mathcal{F}$ at $P$. So the dimensional type is $\tau=e$.

- For all $j \in A_{\text {nd }}$ we have $\nu\left(a_{j}\right) \geq 1$. In this case there is an index $i \notin$ $A$ such that $b_{i}=a_{i}$ is a unit. The vector fields

$$
\xi_{k}=b_{k} \frac{\partial}{\partial x_{i}}-b_{i} \frac{\partial}{\partial x_{k}}, \text { for } k \notin A_{\text {nd }} \cup\{i\},
$$

trivialize $\mathcal{F}$ at $P$. In this case $\tau=e+1$.

In case II of Definition 4 , the trivializing vector fields may be obtained thanks to the integrability condition. Details can be found in Lemma 5 of $[3]$. 
We say that a pre-simple point $P$ is a corner point relatively to $(\mathcal{F}, D)$ if $\tau=e$. If $\tau=e+1$ we say that $P$ is a trace point relatively to $(\mathcal{F}, D)$.

Theorem 1 ([3, Proposition 46]). Let $P$ be a pre-simple singularity for $(\mathcal{F}, D)$ of dimensional type $\tau$. There are formal coordinates $\left(\hat{x}_{1}, \ldots, \hat{x}_{n}\right)$ adapted to $D$ such that

$$
D_{\mathrm{nd}} \subset \bigcup_{i=1}^{\tau}\left(\hat{x}_{i}=0\right),
$$

and $\mathcal{F}$ is given locally at $P$ by a formal equation $\omega=0$ of one of the following types:

A. There are $\lambda_{i} \in \mathbb{C}^{*}, i=1, \ldots, \tau$ such that

$$
\omega=\sum_{i=1}^{\tau} \lambda_{i} \frac{d \hat{x}_{i}}{\hat{x}_{i}} .
$$

B. There are integers $k$, with $1 \leq k \leq \tau, p_{1}, \ldots, p_{k} \in \mathbb{Z}_{>0}$, a formal series $\psi \in \mathbb{C}[[t]]$ with $\psi(0)=0$, and complex numbers $\alpha_{2}, \ldots, \alpha_{\tau}$ with $\alpha_{i} \neq 0$ for $i=k+1, \ldots, \tau$, and not all zero if $k=\tau$, such that

$$
\omega=\sum_{i=1}^{k} p_{i} \frac{d \hat{x}_{i}}{\hat{x}_{i}}+\psi\left(\hat{x}_{1}^{p_{1}} \cdots \hat{x}_{k}^{p_{k}}\right) \sum_{i=2}^{\tau} \alpha_{i} \frac{d \hat{x}_{i}}{\hat{x}_{i}} .
$$

C. There are integers $k$, with $2 \leq k \leq \tau, p_{2}, \ldots, p_{k} \in \mathbb{Z}_{>0}$, and complex numbers $\alpha_{2}, \ldots, \alpha_{\tau}$ with $\alpha_{i} \neq 0$ for $i=k+1, \ldots, \tau$, and not all zero if $k=\tau$, such that

$$
\omega=d \hat{x}_{1}-\hat{x}_{1} \sum_{i=2}^{k} p_{i} \frac{d \hat{x}_{i}}{\hat{x}_{i}}+\hat{x}_{2}^{p_{2}} \cdots \hat{x}_{k}^{p_{k}} \sum_{i=2}^{\tau} \alpha_{i} \frac{d \hat{x}_{i}}{\hat{x}_{i}} .
$$

Definition 6. The vector $\boldsymbol{\beta}$ defined by

$$
\boldsymbol{\beta}:= \begin{cases}\boldsymbol{\lambda}=\left(\lambda_{1}, \ldots, \lambda_{\tau}\right) & \text { in case } \mathrm{A}, \\ \boldsymbol{\alpha}=\left(\alpha_{k+1}, \ldots, \alpha_{\tau}\right) & \text { in case } \mathrm{B},\end{cases}
$$

is called residual vector of the normal form.

Remark 5. If $P$ is a corner point, we only can have the types $\mathrm{A}$ or $\mathrm{B}$ of the theorem, and in this case $\omega$ is a formal adapted generator for $\mathcal{F}$. For trace points, the three types are possible. The type $\mathrm{C}$ provides an adapted generator for $\mathcal{F}$. In the cases $A$ and $B$, in order to adapt $\omega$ to the divisor, we have to multiply $\omega$ by the variable $\hat{x}_{i}$ which does not correspond to a component of the divisor, therefore, $\hat{x}_{i} \omega=0$ is an adapted generator. 
Remark 6. We state this theorem without proof, but it is desirable to sketch some parts of the proof. Suppose that we are in the corner case. The formal coordinates $\hat{\boldsymbol{x}}$ which give us the formal normal forms, are obtained as a limit of convergent coordinates. Starting with convergent coordinates $\boldsymbol{x}$ adapted to $D$, we make coordinate changes $\boldsymbol{x}^{(1)}, \boldsymbol{x}^{(2)}, \ldots$, following a process known as Jordanization. All of these coordinate changes are of the form

$$
x_{i}^{(s+1)}:=x_{i}^{(s)} \cdot u_{i}^{(s)}\left(x_{1}^{(s)}, \ldots, x_{\tau}^{(s)}\right),
$$

where the $u_{i}^{(s)}$ are units. Therefore we have that

$$
\hat{x}_{i}=x_{i} \cdot \hat{u}_{i}\left(x_{1}, \ldots, x_{\tau}\right) \text {, }
$$

where $\hat{u}$ is a formal unit. This implies that locally the components of the divisor $\left(x_{i}=0\right)$ are equal to the formal hypersurfaces $\left(\hat{x}_{i}=0\right)$.

In the case of a trace point, the situation is similar except for at most one of the variables. The difference appears when we have a trace point of type A or B. In this situation the convergent coordinates give us $\tau-1$ invariant hypersurfaces, but the formal coordinates show one more germ of formal invariant hypersurface. Suppose that locally $D=$ $\cup_{i=2}^{\tau}\left(x_{i}=0\right)$. First, it is shown that there is an additional germ of formal invariant hypersurface with equation

$$
\hat{x}_{1}:=x_{1}+\hat{\varphi}\left(x_{2}, \ldots, x_{\tau}\right)=0 \text {, }
$$

where $\hat{\varphi}$ is a formal series vanishing at the origin. Now the process is exactly like in the corner case, but replacing $x_{1}$ by $\hat{x}_{1}$.

Let us recall the case of foliations on surfaces studied by Seidenberg in $[\mathbf{9}]$ (see [5] for a more detailed study). Let $\mathcal{F}$ be a foliation given locally at a point $P$ by $\omega=a d x+b d y$. Consider the vector field $\mathcal{X}=b \frac{\partial}{\partial x}-a \frac{\partial}{\partial y}$ determined by $\omega$. Let $L_{\mathcal{X}}$ be the linear part of $\mathcal{X}$, and $\lambda, \mu \in \mathbb{C}$ its eigenvalues. The point $P$ is a pre-simple singularity if $\lambda$ or $\mu$ are non zero. A pre-simple point $P$ is simple if one of the following conditions holds:

- $\lambda \mu=0$ (saddle-node),

- $\lambda \mu \neq 0$ and $\lambda / \mu \notin \mathbb{Q}_{>0}$.

If $\lambda / \mu \notin \mathbb{Q}_{>0}$ we say that the pair $(\lambda,-\mu)$ is non-resonant. The following definitions generalize this notion to arbitrary dimension. 
Definition 7. Let $\boldsymbol{\beta}$ be an s-tuple $\boldsymbol{\beta}=\left(\beta_{1}, \ldots, \beta_{s}\right) \in \mathbb{C}^{s}$. An s-tuple of nonnegative integers $\boldsymbol{m}=\left(m_{1}, \ldots, m_{s}\right) \in \mathbb{Z}_{\geq 0}^{s}$ is a resonance of $\boldsymbol{\beta}$ if

$$
\langle\boldsymbol{m}, \boldsymbol{\beta}\rangle=\sum_{i=1}^{s} m_{i} \beta_{i}=0 .
$$

We say that $\boldsymbol{\beta}$ is non-resonant if its only resonance is $\boldsymbol{m}=\mathbf{0}$.

We use the formal normal forms given by Theorem 1 in order to define simple singularities.

Definition 8. Let $P$ be a pre-simple singularity for $(\mathcal{F}, D)$. It is a simple sigularity if it has a formal normal form of type A or B, and the residual vector is non-resonant.

\section{Stratification of Sing $\mathcal{F}$}

Let $P$ be a point and $\boldsymbol{x}=\left(x_{1}, \ldots, x_{n}\right)$ a local coordinate system adapted to $D$ defined in a neighborhood $U$ of $P$. For all $I \subset\{1, \ldots, n\}$ denote

$$
\begin{aligned}
& S_{I}^{x}:=\left\{Q \in U: Q \in\left(x_{i}=0\right) \forall i \in I, Q \notin\left(x_{j}=0\right) \forall j \notin I\right\}, \\
& T_{I}^{x}:=\overline{S_{I}^{x}}=\left\{Q \in U: Q \in\left(x_{i}=0\right) \forall i \in I\right\} .
\end{aligned}
$$

Theorem 1 gives a formal description of the singular locus $\operatorname{Sing} \mathcal{F}$ around a pre-simple point for $(\mathcal{F}, D)$. In adapted formal coordinates $\hat{\boldsymbol{x}}=$ $\left(\hat{x}_{1}, \ldots, \hat{x}_{n}\right)$ we have

$$
\begin{array}{ll}
\text { Sing } \mathcal{F}=\bigcup_{1 \leq i<j \leq \tau} T_{i, j}^{\hat{x}}, & \text { if } P \text { is of type A or B, } \\
\text { Sing } \mathcal{F}=\bigcup_{2 \leq i \leq k} T_{1, i}^{\hat{x}} \cup \bigcup_{2 \leq i<j \leq \tau} T_{i, j}^{\hat{x}}, & \text { if } P \text { is of type C. }
\end{array}
$$

We have that this formal description is in fact convergent. We present this result in the following proposition, whose proof follows from Remark 6.

Proposition 3. Let $P$ be a pre-simple point for $(\mathcal{F}, D)$ of dimensional type $\tau$. There are local coordinates $\boldsymbol{x}=\left(x_{1}, \ldots, x_{n}\right)$ adapted to $D$ such that

$$
\begin{array}{ll}
\text { Sing } \mathcal{F}=\bigcup_{1 \leq i<j \leq \tau} T_{i, j}^{x}, & \text { if } P \text { is of type } A \text { or } B, \\
\text { Sing } \mathcal{F}=\bigcup_{2 \leq i \leq k} T_{1, i}^{\boldsymbol{x}} \cup \bigcup_{2 \leq i<j \leq \tau} T_{i, j}^{\boldsymbol{x}}, & \text { if } P \text { is of type } C
\end{array}
$$

locally at $P$, and such that the vector fields $\partial / \partial x_{\tau+1}, \ldots, \partial / \partial x_{n}$ are tangent to $\mathcal{F}$. 
Denote

$\operatorname{Sing}^{*}(\mathcal{F}, D):=\{P \in M \mid P$ is not pre-simple for $(\mathcal{F}, D)\}$.

Proposition 4. $\operatorname{Sing}^{*}(\mathcal{F}, D)$ is a closed set.

Proof: Proposition 14 of [3].

From now on, we assume that $\operatorname{Sing}^{*}(\mathcal{F}, D)=\emptyset$.

Consider the sets

$M_{s}:=\{P \in M \mid$ the dimensional type of $\mathcal{F}$ at $P$ is at least $s\}$,

for $s=1, \ldots, n$. By Proposition 1 all these sets are closed. Note that in particular Sing $\mathcal{F}=M_{2}$.

Proposition 5. The set $M_{s}$ is a finite union of irreducible components of codimension $s$ with normal crossings with $D$. In addition, all points of a connected component of $M_{s} \backslash M_{s+1}$ are analytically equivalent.

Proof: Let $P \in M_{s} \backslash M_{s+1}$ and let $\boldsymbol{x}$ be a local coordinate system adapted to $D$. By Proposition 3 we have that the points of $T_{1, \ldots, \tau}^{\boldsymbol{x}}$ are analytically equivalent to $P$ (the analytic triviality is given by the flow of the vector fields $\left.\partial / \partial x_{\tau+1}, \ldots, \partial / \partial x_{n}\right)$, and outside this set the dimensional type decreases.

By Proposition 3 we have that the sets $M_{s}$ and $D$ locally have normal crossings. Note that each subvariety $T_{I}^{\boldsymbol{x}}$ is contained in different components of the divisor, consequently, they are all different from each other. From this fact follows the global normal crossings.

Remark 7. If $P$ is a point of dimensional type $\tau$, and $\boldsymbol{x}$ is a local coordinate system adapted to $D$, then

$$
M_{r}=\bigcup_{\substack{\# I=r \\ I \subset\{1, \ldots, \tau\}}} S_{I}^{\boldsymbol{x}},
$$

locally at $P$.

Remark 8. Note that if $\operatorname{Sing}^{*}(\mathcal{F}, D) \neq \emptyset$ the proposition is false. Consider for example the foliation of $\mathbb{C}^{3}$ given by $d(x y(x-y)(x-z y))=0$. In this case $M_{3}=(x=y=0)$ has codimension two.

We have that $\operatorname{Sing} \mathcal{F}$ is the union of finitely many codimension two regular subvarieties. Let $\operatorname{Tr} \operatorname{Sing} \mathcal{F} \subset \operatorname{Sing} \mathcal{F}$ be the set of all the trace points. As we shall precise below, we know that $\operatorname{TrSing} \mathcal{F}$ is the union of some irreducible components of Sing $\mathcal{F}$ that we call trace components. The next proposition is due to Cano and Cerveau. 
Proposition 6. If all points are simple, each connected component of TrSing $\mathcal{F}$ defines the germ of a formal invariant hypersurface.

Proof: See [4, Part II, §5].

Remark 9. We are not supposing that all points are simple, therefore we cannot use Proposition 6. If all points are pre-simple, we do not have a unique germ of formal hypersurface invariant for $\mathcal{F}$, due to its possible dicriticalness. However, the connected components of $\operatorname{TrSing} \mathcal{F}$ are well defined, and this fact is the property that we need.

Proposition 5 allows us to stratify Sing $\mathcal{F}$ considering the connected components of $M_{s} \backslash M_{s+1}$, for $s=2, \ldots, n$, as strata (where $M_{n+1}=\emptyset$ ). Since we are working with a divisor which may have dicritical components, it is useful to add this data to the stratification. Thus, the points of a stratum are characterized by being analytically equivalent and belonging to the same components of the divisor. If we denote by $\tau(\Gamma)$ the dimensional type of the stratum $\Gamma$ and by $d(\Gamma)$ the number of dicritical components which contain it, we have

$$
\operatorname{dim}(\Gamma)=n-\tau(\Gamma)-d(\Gamma)
$$

For simplicity, it is useful to extend the stratification to the whole variety $M$. We just need to add to the stratification some strata of dimensional type 1: one trace stratum for the points outside the divisor, a corner stratum for each non-dicritical component (formed by the regular points), and the strata corresponding to regular points over dicritical components.

We say that the stratum $\Gamma$ has the property $\mathcal{P}$ if that property is satisfied by its points (note that all points of a stratum are analytically equivalent). To describe $\mathcal{F}$ at a stratum $\Gamma$ we use as a local generator one corresponding to an arbitrary point $P \in \Gamma$. From now on we will talk about strata rather than points.

Let $\Gamma$ be a stratum of dimensional type $\tau$ contained in $d$ dicritical components. By Proposition 2, we know that $\Gamma$ can be a corner stratum or a trace one. If it is of corner type, then it is, locally at $\Gamma$, the intersection of $\tau$ non-dicritical components of the divisor and $d$ dicritical ones. However, if $\Gamma$ is a trace stratum, it is the intersection of $\tau-1$ non-dicritical components, one connected component of $\operatorname{TrSing} \mathcal{F}$ and $d$ dicritical components. 


\section{Transforming foliations by non singular blow-ups with codimension two centers}

In this work we only use blow-ups with regular centers of codimension two, which are irreducible components of the singular locus. Denote by $E_{1}, \ldots, E_{t}$ the non-dicritical components of $D$ and by $F_{1}, \ldots, F_{s}$ the connected components of $\operatorname{TrSing} \mathcal{F}$.

Definition 9. A subvariety $Y \subset M$ is an allowed center if it is of the form $E_{i} \cap E_{j}$ (corner centers) or $E_{i} \cap F_{j}$ (trace centers).

Remark 10. Note that there are only two options for a stratum: either it is contained in the center, or they are disjoint.

Let $\pi: M^{\prime} \longrightarrow M$ be the blow-up with center $Y$. In $M^{\prime}$ we have a foliation $\mathcal{F}^{\prime}$ and a divisor $D^{\prime}$ where:

- $\mathcal{F}^{\prime}$ is the foliation of $M^{\prime}$ defined locally in the following way. Let $\Gamma^{\prime} \subset M^{\prime}$ be a stratum and let $\Gamma=\pi\left(\Gamma^{\prime}\right) \in M$ be its image by $\pi$. Let $\omega=0$ be a local equation of $\mathcal{F}$ at $\Gamma$. We have that $\mathcal{F}^{\prime}$ is given locally at $\Gamma^{\prime}$ by $\omega^{\prime}=0$, where $\omega^{\prime}:=\pi^{*}(\omega)$ is the pull-back of $\omega$ by $\pi$.

- $D^{\prime}=\cup_{i=1}^{t+1} D_{i}^{\prime}$, where $D_{i}^{\prime}$ is the strict transform of $D_{i}$ for $i=1, \ldots, t$, and $D_{t+1}^{\prime}=\pi^{-1}(Y)$ is the exceptional divisor.

We need to describe how the stratification is modified by the blow-up.

Proposition 7. Let $\Gamma \subset M$ be a stratum. Let $\pi$ be the blow up with center $Y$. If $\Gamma \not \subset Y$, then there is just one stratum $\Gamma^{\prime} \subset M^{\prime}$ such that $\pi\left(\Gamma^{\prime}\right)=\Gamma$. In this case, $\Gamma^{\prime}$ is analytically equivalent to $\Gamma$. Nevertheless, if $\Gamma \subset Y$, there are three strata in $M^{\prime}$ that are projected by $\pi$ onto $\Gamma$. Denoting these strata by $\Gamma^{1}, \Gamma^{2}$, and $\tilde{\Gamma}$, we have

$$
\begin{gathered}
\operatorname{dim}\left(\Gamma^{1}\right)=\operatorname{dim}\left(\Gamma^{2}\right)=\operatorname{dim}(\Gamma) ; \\
\operatorname{dim}(\tilde{\Gamma})=\operatorname{dim}(\Gamma)+1 .
\end{gathered}
$$

Proof: We use codimension two centers, so it is useful to recall here the dimension two case. Let us consider the plane with the divisor formed by both coordinate axes. The exceptional divisor obtained after blowing-up the origin is a projective line. We can divide this line into three subsets: the origin of each chart (defined by the intersection between the exceptional divisor and each component of the strict transform of the divisor), and the rest of the points. The blow-up induces an isomorphism between the punctured plane and the new variety without the exceptional divisor.

Without loss of generality, suppose that $Y$ is a corner center, say $Y=E_{i} \cap E_{j}$ (for trace centers the reasoning is the same). 
Outside the center, the blow-up is a birational morphism, thus, the strata disjoint from the center are not modified, and they are in one to one correspondence with the strata of $M^{\prime} \backslash \pi^{-1}(Y)$.

Let $\Gamma$ be a stratum contained in $Y$. We have that, as sets,

$$
\pi^{-1}(\Gamma) \simeq \Gamma \times \mathbb{P}^{1} .
$$

As in the two dimensional case described above, divide $\pi^{-1}(\Gamma)$ in three subsets:

$$
\begin{aligned}
\Gamma^{1} & :=\pi^{-1}(\Gamma) \cap E_{i} ; \\
\Gamma^{2} & :=\pi^{-1}(\Gamma) \cap E_{j} ; \\
\tilde{\Gamma} & :=\pi^{-1}(\Gamma) \backslash\left(\Gamma^{1} \cup \Gamma^{2}\right) .
\end{aligned}
$$

The subset $\Gamma^{1}$ (respectively $\Gamma^{2}$ ) corresponds to the origin of the first (respectively second) chart. It is contained in $E_{i}$ (respectively $E_{j}$ ) and in the exceptional divisor, but it is disjoint to $E_{j}$ (respectively $E_{i}$ ). The remaining points of $\pi^{-1}(\Gamma)$ are only contained in the exceptional divisor.

The analytic triviality along these subsets can be obtained by lifting the corresponding diffeomorphisms for $\Gamma$.

Remark 11. Note that it does not matter if the blow-up is dicritical or not, these sets are always well defined. In fact, this is the reason to consider the dicritical components in the stratification.

Let $\left(x_{1}, \ldots, x_{n}\right)$ be a coordinate system adapted to $D$ at $\Gamma$. Let $\omega$ be a local generator of $\mathcal{F}$ adapted to $D$ at $\Gamma$. Suppose that locally we have $E_{s}=\left(x_{i}=0\right)$ and $E_{t}=\left(x_{j}=0\right)$. To obtain the expression of $\pi^{*}(\omega)$ we just need to substitute the equations of the blow-up in the expression of $\omega$. In the first chart we have that

$$
\begin{aligned}
x_{i}=x_{i}^{\prime} & \Rightarrow d x_{i}=d x_{i}^{\prime}, \\
x_{j}=\left(x_{j}^{\prime}+\epsilon\right) x_{i}^{\prime} & \Rightarrow d x_{j}=x_{i}^{\prime} d x_{j}^{\prime}+\left(x_{j}^{\prime}+\epsilon\right) d x_{i}^{\prime},
\end{aligned}
$$

where $\epsilon$ depends on the point we are looking at. The value $\epsilon=0$ corresponds to $\Gamma^{1}$, and the different values $\epsilon \neq 0$ correspond to the points of $\tilde{\Gamma}$. Interchanging the indices $i$ and $j$ we obtain the equations for the second chart (thus for $\Gamma^{2}$ ).

Proposition 8. Let $\Gamma \subset Y$ be a simple stratum. Then $\pi$ is non-dicritical and $\Gamma^{1}, \Gamma^{2}$, and $\tilde{\Gamma}$ are simple strata.

Proof: Suppose that $\Gamma$ is a stratum of type A, and fix adapted coordinates $\boldsymbol{x}$ such that $Y=T_{i, j}$. Let $\boldsymbol{\lambda}$ be the residual vector of $\Gamma$. A direct calculation shows that $\Gamma^{1}$ is a type A stratum whose residual vector is 
$\lambda^{\prime}:=\lambda+\lambda_{j} \boldsymbol{e}_{i}$ (where $\boldsymbol{e}_{i}$ is the $\mathrm{i}$-th vector of the canonical basis). Let $\boldsymbol{p}^{\prime} \in \mathbb{Z}^{\tau}$ be a vector orthogonal to $\boldsymbol{\lambda}^{\prime}$. We have that $\boldsymbol{p}:=\boldsymbol{p}^{\prime}+\boldsymbol{p}_{i}^{\prime} \boldsymbol{e}_{j}$ is orthogonal to $\boldsymbol{\lambda}$. If $\boldsymbol{p}^{\prime} \in \mathbb{Z}_{\geq 0}^{\tau}$, then $\boldsymbol{p} \in \mathbb{Z}_{\geq 0}^{\tau}$ and therefore $\boldsymbol{\lambda}^{\prime}$ is nonresonant. This reasoning works for the other strata, and for the case of a type B singularity.

\section{Statement of the main theorem}

Let us make a precise statement of the result of reduction to simple singularities:

Theorem 2. Let $M$ be a germ around a non singular compact analytic subset of a complex analytic variety of dimension $n, D \subset M$ a divisor with normal crossings, and $\mathcal{F}$ a foliation of $M$. Assume that all points of $M$ are pre-simple for $(\mathcal{F}, D)$. There is a finite sequence of blow-ups

$$
M=M_{0} \stackrel{\pi_{1}}{\longleftarrow} M_{1} \stackrel{\pi_{2}}{\longleftarrow} \cdots \stackrel{\pi_{N}}{\longleftarrow} M_{N}=M^{\prime}
$$

satisfying:

- the center $Y_{i}$ of $\pi_{i+1}$ is an irreducible component of $\operatorname{Sing} \mathcal{F}_{i}$, where $\mathcal{F}_{i}$ is the transformed foliation of $\mathcal{F}$ in $M_{i}$,

- $D_{i}:=\left(\pi_{1} \circ \cdots \circ \pi_{i}\right)^{-1}(D) \subset M_{i}$ is a normal crossings divisor,

such that all points of $M^{\prime}$ are simple for $\left(\mathcal{F}^{\prime}, D^{\prime}\right)$.

We divide the proof in two parts. First we eliminate the type $\mathrm{C}$ singularities (Theorem 3) and after this we eliminate the resonances (Theorem 4).

\section{Elimination of type $\mathrm{C}$ singularities}

In the two dimensional case, type $\mathrm{C}$ points are completely characterized by their behavior under blow-up. We recall here this case since it is very similar to the arbitrary dimension case. Consider the foliation of $\mathbb{C}^{2}$ given by

$$
\omega=d x-p x \frac{d y}{y}+y^{p} \alpha \frac{d y}{y},
$$

and suppose that $p>1$. If we blow-up the origin, we obtain two singularities in the exceptional divisor: a saddle node in the origin of the first chart and a type $\mathrm{C}$ singularity in the origin of the second chart. Replacing $p$ by $p-1$ in the above expression we obtain a local generator at the new type $\mathrm{C}$ point. After blowing-up $p-1$ times we obtain one type $\mathrm{C}$ singularity with $p=1$. If we blow-up one more time, the only singularity that appears is a saddle node in the origin of the first chart. 
As illustrated by the two dimensional case, if we blow-up type $\mathrm{C}$ points enough times, they disappear. Our strategy for eliminating all type $\mathrm{C}$ points consists in blowing-up all the trace components of the singular locus which contains type $\mathrm{C}$ strata.

Proposition 9. Let $\pi$ be the blow-up of $M$ with allowed center $Y$. Each type $C$ stratum of $M$ produces at most one type $C$ stratum in $M^{\prime}$.

Proof: As Proposition 7 states, there is nothing to do for strata disjoint from $Y$. Using again Proposition 7 we know that there are three strata over the ones contained in the center. Using the same arguments as in Proposition 8 we see that type A and B strata only produce the same kind of strata (or even non singular strata). So, if there are no type $\mathrm{C}$ strata contained in $Y$, the statement is true.

Suppose that $\Gamma \subset Y$ is a type $\mathrm{C}$ stratum of dimensional type $\tau$. We can take adapted coordinates $\hat{\boldsymbol{x}}$ such that locally $Y=T_{1, k}^{\hat{\boldsymbol{x}}}, \Gamma=S_{1, \ldots, \tau}^{\hat{\boldsymbol{x}}}$ and such that

$$
\omega=d \hat{x}_{1}-\hat{x}_{1} \sum_{i=2}^{k} p_{i} \frac{d \hat{x}_{i}}{\hat{x}_{i}}+\hat{x}_{2}^{p_{2}} \cdots \hat{x}_{k}^{p_{k}} \sum_{i=2}^{\tau} \alpha_{i} \frac{d \hat{x}_{i}}{\hat{x}_{i}}
$$

is a local generator of $\mathcal{F}$ at $\Gamma$. Denote by $\Delta^{1}, \Delta^{2}$, and $\tilde{\Delta}$ the strata that locally are equal to $S_{2, \ldots, \tau}^{\hat{\boldsymbol{x}}}, S_{1, \ldots, k-1, k+1, \ldots, \tau}^{\hat{\boldsymbol{x}}}$, and $S_{2, \ldots, k-1, k+1, \ldots, \tau}^{\hat{\boldsymbol{x}}}$ respectively. We have that $\Gamma$ is contained in $\overline{\Delta^{i}}(i=1,2)$ and in $\overline{\tilde{\Delta}}$. Depending on the values of $\tau, k, p_{k}$, and $\alpha_{k}$ the behavior of $\Gamma$ under the blow-up centered at $Y$ is different. First, suppose that $\tau \geq 3$.

- If $p_{k}>1$ the blow-up is non-dicritical. Thus, $\tilde{\Gamma}$ and $\Gamma^{1}$ are corner strata and consequently they cannot be of type C. Replacing $p_{k}$ by $p_{k}-1$ in (5) we obtain a local generator at $\Gamma^{2}$.

- If $p_{k}=1$ and $\alpha_{k} \neq 0$ the blow-up is also non-dicritical. The situation is like in the previous case. Note that now $p_{k}-1=0$, and therefore the index $k$ drops to $k-1$.

- If $p_{k}=1$ and $\alpha_{k}=0$ the blow-up is dicritical. The points of $\Gamma^{1}$, $\Gamma^{2}$, and $\tilde{\Gamma}$ are analytically equivalent to the ones of $\Delta^{1}, \Delta^{2}$, and $\tilde{\Delta}$ respectively, but they belong to one more dicritical component of the divisor. Note that in the three strata the dimensional type has dropped. Replacing $p_{k}$ by $p_{k}-1=0$ in the expression (5) we obtain a local generator for $\Gamma^{2}$, and this is the unique type $\mathrm{C}$ stratum created.

If $\tau=2$ we cannot have the third case (if $\alpha_{2}=0$ then $\Gamma$ is a trace stratum of type A). In the other cases the situation is similar, but note that if the index $k$ becomes $0, \Gamma^{2}$ is a non singular stratum. 
Theorem 3. Let $M$ be a germ around a non singular compact analytic subset of a complex analytic variety of dimension $n, D \subset M$ a divisor with normal crossings, and $\mathcal{F}$ a foliation of $M$. Assume that all points of $M$ are pre-simple for $(\mathcal{F}, D)$. There is a finite sequence of blow-ups

$$
M=M_{0} \stackrel{\pi_{1}}{\longleftarrow} M_{1} \stackrel{\pi_{2}}{\longleftarrow} \cdots \stackrel{\pi_{N}}{\longleftarrow} M_{N}=M^{\prime}
$$

satisfying:

- the center $Y_{i}$ of $\pi_{i+1}$ is an irreducible component of Sing $\mathcal{F}_{i}$, where $\mathcal{F}_{i}$ is the transformed foliation of $\mathcal{F}$ in $M_{i}$,

- $D_{i}:=\left(\pi_{1} \circ \cdots \circ \pi_{i}\right)^{-1}(D) \subset M_{i}$ is a normal crossings divisor,

such that there are no type $C$ points in $M^{\prime}$.

Proof: For each stratum $\Gamma$ consider the invariant $\iota(\Gamma) \in \mathbb{Z}_{\geq 0}^{3}$ defined by

$$
\iota(\Gamma):= \begin{cases}\left(1, k, p_{2}+\cdots+p_{k}\right) & \text { if } \Gamma \text { is a type } \mathrm{C} \text { stratum } \\ (0,0,0) & \text { otherwise }\end{cases}
$$

where the numbers $k$ and $p_{2}+\cdots+p_{k}$ are exactly the ones that appear in a formal normal form.

Let $\pi$ be the blow-up of $M$ with allowed center $Y$ of trace type. By Proposition 9 we know that there is at most one type $\mathrm{C}$ stratum in $M^{\prime}$ over a stratum of $M$. Then, for each type $\mathrm{C}$ stratum $\Gamma^{\prime}$ of $M^{\prime}$ there is one stratum $\Gamma$ in $M$ such that $\pi\left(\Gamma^{\prime}\right)=\Gamma$. As we can see in the proof of Proposition 9 we have that

$$
\iota\left(\Gamma^{\prime}\right) \leq \iota(\Gamma)
$$

for the lexicographic order. Moreover, the inequality is strict for strata contained in the center of the blow-up.

There are finitely many strata of type C. Number them by $\Gamma_{1}, \ldots, \Gamma_{N}$ and consider the global invariant

$$
\iota_{C}:=\sum_{i=1}^{N} \iota\left(\Gamma_{i}\right)
$$

Each time we blow-up an allowed trace center containing type C strata, the invariant $\iota_{C}$ decreases for the lexicographic order. Consequently, after a finite chain of such blow-ups there will not be any type $\mathrm{C}$ stratum. Therefore the strategy consists in blowing-up all the allowed trace centers containing type $\mathrm{C}$ strata. We can do this by choosing the centers arbitrarily, or, for example, taking each time one which contains a stratum that reaches the maximum value of the invariant. 


\section{Elimination of resonances}

After eliminating all type $\mathrm{C}$ singularities, the only non simple strata are resonant of type $\mathrm{A}$ or $\mathrm{B}$. The following proposition allows us to know when a stratum is resonant without using the formal normal forms.

Proposition 10. Let $\Gamma$ be a pre-simple stratum of type $A$ or $B$. The following conditions are equivalent:

(1) $\Gamma$ is resonant.

(2) There is a finite chain of blow-ups

$$
M=M_{0} \stackrel{\pi_{1}}{\longleftarrow} M_{1} \stackrel{\pi_{2}}{\longleftarrow} \cdots \stackrel{\pi_{N}}{\longleftarrow} M_{N}=M^{\prime}
$$

satisfying:

- The center $Y_{i}$ of $\pi_{i+1}$ is an irreducible component of $\operatorname{Sing} \mathcal{F}$, and there is a stratum $\Gamma_{i} \subset Y_{i}$, such that $\operatorname{dim}\left(\Gamma_{i}\right)=\operatorname{dim}(\Gamma)$ and such that $\pi_{1} \circ \cdots \circ \pi_{i}\left(\Gamma_{i}\right)=\Gamma$.

- $\pi_{N}$ is dicritical, and it is the only one in the chain with this property.

Proof: Let $\boldsymbol{\lambda}$ be the residual vector of $\Gamma$. If $\Gamma$ is a type $B$ stratum we only use centers of blow-up involving the variables which give the residual vector. Thus we can think without loss of generality that $\Gamma$ is a type $\mathrm{A}$ stratum.

(1) $\Rightarrow(2)$ Let $\boldsymbol{r}^{0}=\left(r_{1}, \ldots, r_{\tau}\right)$ be a resonance of $\Gamma^{0}:=\Gamma$ such that $\operatorname{gcd}\left(r_{1}, \ldots, r_{\tau}\right)=1$. Fix two indices $i, j$ with $r_{i}, r_{j}>0$. Let $Y_{0}$ be the subvariety locally equal to $T_{i, j}^{\boldsymbol{x}}$, and let $\pi$ be the blow-up centered at $Y_{0}$. If $r_{i} \leq r_{j}$ then the stratum $\Gamma^{1}$ has $\boldsymbol{r}^{1}=\boldsymbol{r}^{0}-r_{i} \boldsymbol{e}_{j}$ as resonance. If instead we have $r_{i}>r_{j}$ the stratum $\Gamma^{2}$ has $\boldsymbol{r}^{1}=\boldsymbol{r}^{0}-r_{j} \boldsymbol{e}_{i}$ as resonance. Take $\Gamma_{1}$ equal to one of the resonant strata and denote by $\boldsymbol{r}^{1}$ its resonance. We have that $\left|\boldsymbol{r}^{1}\right|<\left|\boldsymbol{r}^{0}\right|$. We can repeat this process until we reach a stratum $\Gamma^{N-1}$ with resonance $\boldsymbol{r}^{N-1}=\boldsymbol{e}_{s}+\boldsymbol{e}_{t}$. Let $Y_{N-1}$ be the subvariety locally equal to $T_{s, t}^{\boldsymbol{x}}$, and let $\pi_{N}$ be the blow-up centered at $Y_{N-1}$. This last blow-up is dicritical and therefore the described sequence of blow-ups is the desired chain.

$(2) \Rightarrow(1)$ We need to construct a resonance of $\Gamma$ using the chain of blow-ups (6). We know that $\boldsymbol{r}^{N-1}=\boldsymbol{e}_{s}+\boldsymbol{e}_{t}$ is a resonance of $\Gamma_{N-1}$ since $\pi_{N}$ is dicritical. The stratum $\Gamma_{N-1}$ is in the origin of one of the charts of the blow-up $\pi_{N-1}$. If for example, it is in the first chart, we have that $\boldsymbol{r}^{N-2}=\boldsymbol{r}^{N-1}+r_{i}^{N-1} \boldsymbol{e}_{j}$ is a resonance for $\Gamma_{N-2}$. Iterating this process we get a resonance for $\Gamma$. 
Proposition 11. Let $\Gamma$ and $\Delta$ be strata of $M$. If $\Delta \subset \bar{\Gamma}$ and $\Gamma$ is resonant, then $\Delta$ is also resonant.

Proof: By Proposition 10 we know that there is a finite chain of blow-ups satisfying certain conditions related to $\Gamma$. The same sequence of blowups satisfies the same conditions with respect to $\Delta$. Using again the equivalence of the previous proposition we have that $\Delta$ is resonant.

Proposition 12. Suppose that all type A strata are non-resonant. Then, all type $B$ strata are also non-resonant.

Proof: Let $\Gamma$ be a type B stratum and suppose that it is resonant. Let $\hat{\boldsymbol{x}}$ be an adapted to $D$ coordinate system such that $\Gamma$ is locally equal to $S_{1, \ldots, \tau}^{\hat{\boldsymbol{x}}}$ and let

$$
\omega=d \hat{x}_{1}-\hat{x}_{1} \sum_{i=2}^{k} p_{i} \frac{d \hat{x}_{i}}{\hat{x}_{i}}+\hat{x}_{2}^{p_{2}} \cdots \hat{x}_{k}^{p_{k}} \sum_{i=2}^{\tau} \alpha_{i} \frac{d \hat{x}_{i}}{\hat{x}_{i}}
$$

be a formal normal form. The residual vector of $\Gamma$ is $\boldsymbol{\lambda}=\left(\alpha_{k+1}, \ldots, \alpha_{\tau}\right)$. Let $\boldsymbol{r}$ be a resonance of $\Gamma$ with the maximum amount of zeros and let $I \subset\{k+1, \ldots, \tau\}$ be the set of indices $I:=\left\{i \mid r_{i} \neq 0\right\}$. Let $\Delta$ be the stratum locally equal to $S_{I}^{\boldsymbol{x}}$. Using the arguments of the proof of Proposition 10 we can determine a chain of blow-ups in the conditions of the proposition involving only the variables corresponding to $I$. This chain of blow-ups also satisfies the conditions with respect to $\Delta$. Thus, by Proposition 10, we have that $\Delta$ is resonant. This stratum is resonant involving all the variables, and therefore it is of type A contradicting the hypothesis.

Theorem 4. Let $M$ be a germ around a non singular compact analytic subset of a complex analytic variety of dimension $n, D \subset M$ a divisor with normal crossings, and $\mathcal{F}$ a foliation of $M$. Assume that all points of $M$ are pre-simple for $(\mathcal{F}, D)$, and that there are no type $C$ points. There is a finite sequence of blow-ups

$$
M=M_{0} \stackrel{\pi_{1}}{\longleftarrow} M_{1} \stackrel{\pi_{2}}{\longleftarrow} \cdots \stackrel{\pi_{N}}{\longleftarrow} M_{N}=M^{\prime}
$$

satisfying

- the center $Y_{i}$ of $\pi_{i+1}$ is an irreducible component of $\operatorname{Sing} \mathcal{F}_{i}$, where $\mathcal{F}_{i}$ is the transformed foliation of $\mathcal{F}$ in $M_{i}$,

- $D_{i}:=\left(\pi_{1} \circ \cdots \circ \pi_{i}\right)^{-1}(D) \subset M_{i}$ is a normal crossings divisor, such that all points of $M^{\prime}$ are simple for $\left(\mathcal{F}^{\prime}, D^{\prime}\right)$.

The idea is to reduce the problem to a combinatorial process of elimination of indeterminacies. First we need to treat all the strata together, 
both the trace and the corner ones. Then we define the Control Variety: a variety with a divisor which represents the properties of $\operatorname{Sing} \mathcal{F}$ that we need. In this variety we define and solve a combinatorial game. Finally we show that this solves also the problem of eliminating resonances.

As we saw in the previous sections, the irreducible components of Sing $\mathcal{F}$ are determined by $E_{1}, \ldots, E_{t}$ (the non-dicritical components of the divisor) and $F_{1}, \ldots, F_{s}$ (the connected components of $\operatorname{Tr} \operatorname{Sing} \mathcal{F}$ ). Rename these sets by $\tilde{K}_{1}, \ldots, \tilde{K}_{N}$, where $N=s+t, \tilde{K}_{i}=E_{i}$ for $i=$ $1, \ldots, t$, and $\tilde{K}_{t+j}=F_{j}$ for $j=1, \ldots, s$. Denote by $\tilde{\mathcal{K}}$ the set $\tilde{\mathcal{K}}:=$ $\left\{\tilde{K}_{1}, \ldots, \tilde{K}_{N}\right\}$.

In order to treat all the strata together, we will represent all the residual vectors and resonances in a common space. Let $\Gamma$ be a type A strata of dimensional type $\tau$. Note that $\Gamma$ is contained in exactly $\tau$ elements of $\tilde{\mathcal{K}}$. Let $I(\Gamma) \subset\{1, \ldots, N\}$ be the set of indices corresponding to these elements. Let $\boldsymbol{\lambda} \in\left(\mathbb{C}^{*}\right)^{\tau}$ be the residual vector of $\Gamma$. Each coefficient $\lambda_{i}$ corresponds to one of the components of $\tilde{\mathcal{K}}$, say $\tilde{K}_{n_{i}}$. Consider the injective map

$$
\iota:\left(\mathbb{C}^{*}\right)^{\tau} \longrightarrow \mathbb{C}^{N}
$$

defined by $\iota(\boldsymbol{\lambda})_{n_{i}}=\lambda_{i}$ and $\iota(\boldsymbol{\lambda})_{j}=0$ if $j \notin I(\Gamma)$. Using the same idea we can represent the resonances $\boldsymbol{r} \in \mathbb{Z}_{>0}^{\tau}$ into $\mathbb{Z}_{>0}^{N}$. For short, we will write $\boldsymbol{\lambda}$ instead of $\iota(\boldsymbol{\lambda})$ (and we do the same for the resonances).

Consider the vector subspace of $\mathbb{Q}^{N}$

$$
\left.\operatorname{Res}(\Gamma):=\left\langle\boldsymbol{r} \in \mathbb{Z}_{\geq 0}^{N} \backslash\{\mathbf{0}\}\right| \boldsymbol{r} \text { is a resonance of } \Gamma\right\rangle_{\mathbb{Q}} .
$$

Note that if $\Gamma$ is non-resonant then $\operatorname{Res}(\Gamma)=\emptyset$. Now consider the subspace of $\mathbb{Q}^{N}$

$$
\left.\operatorname{Res}^{*}(\Gamma):=\left\langle\boldsymbol{p} \in \mathbb{Q}^{N}\right| p_{i}=0 \text { if } i \notin I(\Gamma) \text { and }\langle\boldsymbol{p}, \boldsymbol{r}\rangle=0 \forall \boldsymbol{r} \in \operatorname{Res}(\Gamma)\right\rangle \text {, }
$$

if $\Gamma$ is resonant, and $\operatorname{Res}^{*}(\Gamma)=\emptyset$ otherwise.

Let $B(\Gamma):=\left\{\boldsymbol{p}^{1}, \ldots, \boldsymbol{p}^{t}\right\}$ be a system of generators of $\operatorname{Res}^{*}(\Gamma)$.

Definition 10. Let $\boldsymbol{p} \in \mathbb{Z}^{N}$ be a vector of integers. If $\boldsymbol{p} \in \mathbb{Z}_{\geq 0}^{N}$ or $\boldsymbol{p} \in \mathbb{Z}_{\leq 0}^{N}$, we say that the vector $\boldsymbol{p}$ has pure sign.

Remark 12. Note that a resonance is a vector with pure sign.

Lemma 1. If all the elements of $B(\Gamma)$ have pure sign, then $\Gamma$ is nonresonant.

Proof: By definition, two vectors with pure sign can not be orthogonal. Since the resonances have pure sign, $\Gamma$ has to be non-resonant. 
7.1. The Control Variety. Let $\tilde{A} \in \mathcal{M}_{N \times N}$ be the matrix defined by $\tilde{A}_{i, j}=1$ if $\tilde{K}_{i} \cap \tilde{K}_{j} \neq \emptyset$ and $\tilde{A}_{i, j}=0$ otherwise. We refer to this matrix as the codimension two incidence matrix of $\tilde{\mathcal{K}}$. Let $\left(i_{1}, j_{1}\right)<\cdots<\left(i_{s}, j_{s}\right)$ be the pairs of indices such that $i<j$ and $\tilde{A}_{i, j}=0$, ordered by the lexicographic order.

Consider the affine space $X_{0}=\mathbb{C}^{N}$ and let $\mathcal{K}^{0}=\cup_{i=1}^{N} K_{i}$ be the divisor defined by $K_{i}:=\left(z_{i}=0\right)$, where $\left(z_{1}, \ldots, z_{N}\right)$ are coordinates of $\mathbb{C}^{N}$. Note that the codimension two incidence matrix of $\mathcal{K}^{0}$ is defined by $A^{0}:=\left(A_{i, j}^{0}=1\right)_{i, j=1}^{N}$. We will modify this variety by blow-ups until we get a new one in which the codimension two incidence matrix of the strict transform of the divisor is exactly $\tilde{A}$.

Let $\pi_{1}: X_{1} \longrightarrow X_{0}$ be the blow-up with center $K_{i_{1}} \cap K_{j_{1}}$ and let $\mathcal{K}^{1} \subset X_{1}$ be the strict transform of $\tilde{\mathcal{K}}$ (for simplicity, we denote the strict transform of each component equal to the initial one). Replacing $A_{i_{1}, j_{1}}$ and $A_{j_{1}, i_{1}}$ by 0 we obtain the codimension two incidence matrix of $\tilde{\mathcal{K}}^{1}$. Repeating this process with the pairs $\left(i_{2}, j_{2}\right)<\cdots<\left(i_{s}, j_{s}\right)$, we finally get a projective variety $X:=X_{s}$ and a divisor $\mathcal{K}:=\mathcal{K}^{s}$ whose codimension two incidence matrix is exactly $A:=\tilde{A}$.

7.2. Elimination of local indeterminacies of a divisor. The objects for the combinatorial game mentioned above are divisors with support contained in $\mathcal{K}$. They are all of the form

$$
\Phi:=\sum_{i=1}^{N} n_{i} K_{i}
$$

where $n_{i} \in \mathbb{Z}$. Suppose that we perform the blow up of $X$ centered at $K_{s} \cap K_{t}$. By abuse of notation denote the strict transforms of $K_{1}, \ldots, K_{N}$ with the same symbols, and denote by $K_{N+1}$ the exceptional divisor. We define the strict transform of $\Phi$ as

$$
\Phi^{*}:=\sum_{i=1}^{N+1} n_{i}^{\prime} K_{i},
$$

where $n_{i}^{\prime}=n_{i}$ for $i=1, \ldots, N$ and $n_{N+1}^{\prime}=n_{s}+n_{t}$.

Let $\mu_{i, j}(\Phi)$ be the integer defined by

$$
\mu_{i, j}(\Phi)= \begin{cases}-n_{i} n_{j} & \text { if } i<j, K_{i} \cap K_{j} \neq \emptyset \text { and } n_{i} n_{j}<0, \\ 0 & \text { otherwise. }\end{cases}
$$

Let $M(\Phi)$ be the integer defined by

$$
M(\Phi):=\max _{i, j}\left\{\mu_{i, j}(\Phi)\right\} .
$$


Definition 11. A divisor $\Phi$ is locally determined if $M(\Phi)=0$.

The following lemma is the combinatorial key in our process of elimination of resonances.

Lemma 2. There is a finite chain of blow-ups, all of them centered at the intersection of two components of $\mathcal{K}$, such that the strict transform of $\Phi$ is locally determined.

Proof: Let $l(\Phi)$ be the integer defined by

$$
l(\Phi):=\#\left\{(i, j) \mid i<j \text { and } \mu_{i, j}(\Phi)=M(\Phi)\right\},
$$

and let $\iota(\Phi)$ be the pair

$$
\iota(\Phi):=(M(\Phi), l(\Phi)) .
$$

Choose a pair of indices $(s, t)$ such that $\mu_{s, t}>0$ (suppose without loss of generality that $n_{s}<0<n_{t}$ and $n_{s}+n_{t} \geq 0$ ). Let $\pi$ be the blow-up of $X$ with center $K_{s} \cap K_{t}$. By definition we have that

$$
\mu_{s, t}^{\prime}=0
$$

and

$$
\mu_{i, j}^{\prime}=\mu_{i, j}
$$

if $\{i, j\} \subset\{1, \ldots, N\} \backslash\{s, t\}$. Since $n_{s}<n_{N+1}^{\prime}=n_{s}+n_{t}<n_{t}$ we have that

$$
\mu_{s, N+1}^{\prime}, \mu_{t, N+1}^{\prime}<\mu_{s, t} .
$$

Finally, take an index $i \neq s, t$. If $K_{i} \cap K_{N+1}=\emptyset$, or if $n_{i} \geq 0$ then

$$
\mu_{i, N+1}^{\prime}=0 \text {. }
$$

Otherwise we have

$$
\mu_{i, N+1}^{\prime}=-n_{i}^{\prime} n_{N+1}^{\prime}=-n_{i}\left(n_{s}+n_{t}\right)<-n_{i} n_{t}=\mu_{i, t} .
$$

We have that if the pair $(s, t)$ satisfies $\mu_{s, t}=M(\Phi)$ then

$$
\iota\left(\Phi^{\prime}\right)<\iota(\Phi)
$$

for the lexicographic order. Repeating this process we get $\iota\left(\Phi^{\prime}\right)=0$, hence, $M(\Phi)=0$, as desired.

Remark 13. This lemma is an avatar of the general result of resolution of singularities of Hironaka [7] applied to the case of the elimination of indeterminacies of a rational function that is already locally a quotient of monomials. We take a slightly more general situation, since our divisor is not necessarily a principal divisor. Moreover, our algorithm uses only codimension two centers. In the local uniformization approach 
we are close to the classical results of Zariski [10]; there he uses only codimension two centers.

Definition 12. Let $\Phi=\sum_{i=1}^{N} n_{i} K_{i}$ and $\Psi=\sum_{i=1}^{N} m_{i} K_{i}$ be divisors. We say that $\Psi$ is a subdivisor of $\Phi$ if for all index $i$ we have $m_{i}=n_{i}$ or $m_{i}=0$.

Remark 14. Note that if $\Phi$ is a locally determined divisor, so are all its subdivisors.

7.3. End of the proof of Theorem 4. Suppose that there is just one resonant stratum $\Gamma \subset M$ with $B(\Gamma)=\{\boldsymbol{p}\}$. Consider the divisor $\Phi_{\boldsymbol{p}}$ in $X$ defined by

$$
\Phi_{\boldsymbol{p}}:=\sum_{i=1}^{N} p_{i} K_{i}
$$

Since $\boldsymbol{p}$ has pure sign we have that $\Phi_{\boldsymbol{p}}$ is not locally determined.

Suppose that $\pi$ is the blow-up of $X$ centered at $K_{s} \cap K_{t}$. Let $\Phi_{p}^{*}$ be the strict transform of $\Phi_{\boldsymbol{p}}$ and consider the following subdivisors:

$$
\Phi_{\boldsymbol{p}}^{1}:=\sum_{\substack{i=1 \\ i \neq s}}^{N+1} p_{i} K_{i} ; \quad \tilde{\Phi}_{\boldsymbol{p}}:=\sum_{\substack{i=1 \\ i \neq s, t}}^{N+1} p_{i} K_{i} ; \quad \Phi_{\boldsymbol{p}}^{2}:=\sum_{\substack{i=1 \\ i \neq t}}^{N+1} p_{i} K_{i} .
$$

Let $\tilde{\pi}$ be the blow-up of $M$ centered at $\tilde{K}_{s} \cap \tilde{K}_{t}$. Suppose that $\Gamma^{1}$ is resonant. In the proof of Proposition 8 we saw how to compute the resonances of $\Gamma^{1}$ using the ones related to $\Gamma$. We have that

$$
B\left(\Gamma^{1}\right)=\left\{\boldsymbol{p}^{1}\right\}
$$

where $\boldsymbol{p}^{1}$ is exactly the vector of integers appearing in the expression of $\Phi_{\boldsymbol{p}}^{1}$. Thus, if we associate a divisor to $\boldsymbol{p}^{1}$ we get $\Phi_{\boldsymbol{p}}^{1}$. In the same way, if $\Gamma^{2}$ or $\tilde{\Gamma}$ are resonant, its associated divisors are $\Phi_{p}^{2}$ and $\tilde{\Phi}_{p}$ respectively.

Lemma 2 gives us a finite chain of blow-ups in $X$ which transforms $\Phi_{\boldsymbol{p}}$ into a locally determined divisor. Let $Y_{1}, \ldots, Y_{m}$ be the centers of such blow-ups. Each center is the intersection of two components of $\mathcal{K}$, so we can write $Y_{i}=K_{s_{i}} \cap K_{t_{i}}$ where $\left\{s_{i}, t_{i}\right\} \subset\{1,2, \ldots, N+i-1\}$.

Let $\pi_{1}$ be the blow-up of $M$ with center $\tilde{Y}_{1}=\tilde{K}_{s_{1}} \cap \tilde{K}_{t_{1}}$. We obtain three strata over $\Gamma$, which may be resonant. We need to control these three strata, but as we saw previously, we can do this with subdivisors of $\Phi_{\boldsymbol{p}}^{*}$. The key is in Remark 14: if we transform $\Phi_{\boldsymbol{p}}$ into a locally determined divisor, then all its subdivisors will be also locally determined. Now we can continue the process by blowing-up the center $\tilde{Y}_{2}=\tilde{K}_{s_{2}} \cap \tilde{K}_{t_{2}}$, and so on. In the final step, we have that all the strata over $\Gamma$ are non-resonant. 
In the general case we have finitely many resonant strata. Denote them by $\Gamma_{1}, \ldots, \Gamma_{N}$. Fix systems of generators of $\operatorname{Res}^{*}\left(\Gamma_{i}\right)$

$$
B\left(\Gamma^{i}\right)=\left\{\boldsymbol{p}_{1}^{i}, \ldots, \boldsymbol{p}_{n_{i}}^{i}\right\}
$$

for $i=1, \ldots, N$. Denote by $T$ the number $T=n_{1}+\cdots+n_{N}$ and let $\Phi_{1}, \ldots, \Phi_{T}$ be the divisors related to the elements of $B\left(\Gamma^{i}\right)$.

In the same way we did before, using Lemma 2 we can determine a finite chain of blow-ups which transform $\Phi_{1}$ into a locally determined divisor. This chain of blow-ups transforms also the other divisors. Now, we can use Lemma 2 again so that $\Phi_{\boldsymbol{p}_{2}}$ (its strict transform) becomes locally determined (note that if we transform a locally determined divisor by blow-ups, it stays locally determined). Using Lemma 2 enough times, we determine a chain of blow-ups which makes all the divisors become locally determined. With the corresponding chain of blow-ups in $M$ we reach the situation desired.

Acknowledgements. I would like to express my gratitude to Professor Felipe Cano for bringing the problem to my attention, the useful discussions, suggestions, and revision of the manuscript.

The author was partially supported by the Ministerio de Educación, Cultura y Deporte of Spain (FPU grant AP2010-6042).

\section{References}

[1] C. CAmacho And P. SAD, Invariant varieties through singularities of holomorphic vector fields, Ann. of Math. (2) 115(3) (1982), 579-595. DOI: $10.2307 / 2007013$.

[2] F. CANo, Reduction of the singularities of nondicritical singular foliations. Dimension three, Amer. J. Math. 115(3) (1993), 509-588. DOI: $10.2307 / 2375074$.

[3] F. CANo, Reduction of the singularities of codimension one singular foliations in dimension three, Ann. of Math. (2) 160(3) (2004), 907-1011. DOI: 10.4007/annals.2004.160.907.

[4] F. Cano and D. Cerveau, Desingularization of nondicritical holomorphic foliations and existence of separatrices, Acta Math. 169(1) (1992), 1-103. DOI: $10.1007 /$ BF02392757.

[5] F. Cano, D. Cerveau, and J. DÉserti, "Théorie élémentaire des feuilletages holomorphes singuliers", Collection "Échelles", Éditions Belin, 2013.

[6] F. Cano And J.-F. Mattei, Hypersurfaces intégrales des feuilletages holomorphes, Ann. Inst. Fourier (Grenoble) 42(1-2) (1992), 49-72. 
[7] H. Hironaka, Resolution of singularities of an algebraic variety over a field of characteristic zero. I, II, Ann. of Math. (2) 79 (1964), 109-203; ibid. (2) 79 (1964), 205-326.

[8] J.-F. Mattei And R. Moussu, Holonomie et intégrales premières, Ann. Sci. École Norm. Sup. (4) 13(4) (1980), 469-523.

[9] A. Seidenberg, Reduction of singularities of the differential equation $A d y=B d x$, Amer. J. Math. 90 (1968), 248-269. DOI: $10.2307 / 2373435$.

[10] O. ZARISKI, Local uniformization on algebraic varieties, Ann. of Math. (2) 41 (1940), 852-896. DOI : 10.2307/1968864.

Departamento de Álgebra, Análisis Matemático, Geometría y Topología Universidad de Valladolid

47011 Valladolid

Spain

E-mail address: mfduque@agt.uva.es

Primera versió rebuda el 25 de setembre de 2013, darrera versió rebuda el 22 de novembre de 2013. 